\title{
Structure Within the Local Bubble: Properties of Individual Clouds
}

\author{
J.E. Beckman ${ }^{1}$, J. Trapero ${ }^{1,2,3}$, J.R. Álamo ${ }^{1}$, R. Génova ${ }^{1}$, and I. Lundstrom ${ }^{4}$ \\ 1 Instituto de Astrofísica de Canarias (IAC), La Laguna, Tenerife, Spain \\ 2 LAEFF, Villafranca del Castillo, Madrid, Spain \\ 3 Instituto de Astrofísica de Andalucía, Granada, Spain \\ 4 Lund Astronomical Observatory, Lund, Sweden
}

\section{The Basic Methodology}

Starting from the casual observation that neighbouring lines of sight to stars at similar distances in the LISM show abruptly varying column densities, we developed a technique for measuring properties of single clouds. Fig. 1 shows an idealized version of this. The steps (Trapero et al. 1992, 1995) are:

- Correct for extinction the distances to local stars used, via B-V (where available), or our own measured $\mathrm{NaI}$ or $\mathrm{KI}$ absorptions.

- Measure diametral column density towards $\mathrm{A}$ from $\mathrm{NaI}$ or $\mathrm{KI}$ equivalent width and convert to hydrogen: $\mathrm{N}(\mathrm{H})\left[\mathrm{cm}^{-2}\right]$.

- Measure cloud diameter, between $\mathrm{E}$ and $\mathrm{E}$ '.

- Estimate the number density, $\mathrm{n}(\mathrm{H})=\mathrm{N}(\mathrm{H}) / \mathrm{d}\left[\mathrm{cm}^{-3}\right]$.

- Use a canonical thermal pressure, $\mathrm{nT}=3500 \mathrm{~K}$ cmThis at least gives the decision cool $(<100 \mathrm{~K})$ or warm $(>5000 \mathrm{~K})$.

- Estimate the cloud mass by integrating $\mathrm{n}(\mathrm{H})$ over the estimated volume.

Following up, cool cores within warm clouds, or warm envelopes around cool clouds can be identified using distance and velocity as tags. We have found empirical rules of thumb to guide us:

- The KI resonance at $7699 \AA$ is ideal for finding cool clouds; it is too weak to show up in warm clouds.

- NaI D doublet lines weaker than $5 \mathrm{~m} \AA$ typify warm clouds. There are too many of these, by a factor 50 , to come from cool cloud edges. This is confirmed by the "low velocity Routly-Spitzer effect" (see below).

\section{Examples of Objects Measured}

\subsection{Cool Clouds: a Massive Local Cloud in Perseus}

This section is a brief summary of results in 4 papers (Trapero et al. 1992, 1995, 1996; Serra et al. 1995). Strong HI column densities were inferred towards $\alpha 3 \mathrm{~h} 40 \mathrm{~m}, \delta+48^{\circ}$ via stellar KI absorptions. The above method yielded 


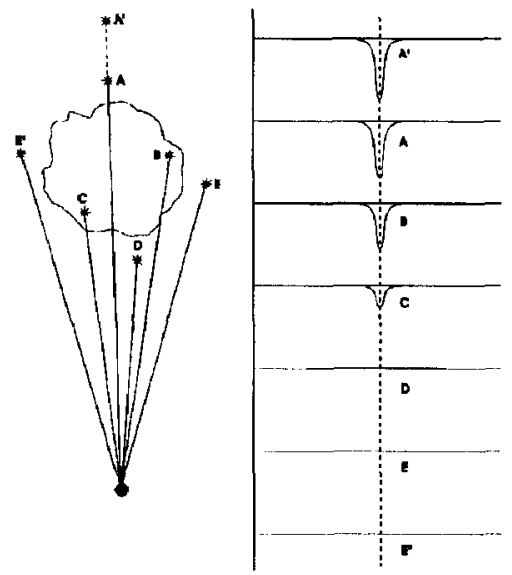

Fig. 1. Schematic showing how the parameters of a LISM cloud are inferred using lines of sight to stars around it (see text).

a cloud mass of $1300 \mathrm{M}_{\odot}$, temperature around $50 \mathrm{~K}$, and density $70 \mathrm{~cm}^{-3}$. Given the velocity, we identified the cloud in absorption against the warmer background, using $21 \mathrm{~cm} \mathrm{HI}$ mapping, revising mass and temperature, and finding minima of $30 \mathrm{~K}$. Molecular cores were then found using $\mathrm{CO}$ mapping. Fig.2 is a map of HI temperature, from $21 \mathrm{~cm}$ data, and Fig. 3 a map of a molecular core, whose mass is only a few $\mathrm{M}_{\odot}$. The whole cloud lies within $120 \mathrm{pc}$ of the sun. Numerous smaller but still massive $\left(\mathrm{M}>200 \mathrm{M}_{\odot}\right)$ clouds have been found via KI; they occupy less than $10 \%$ of the LISM volume, but form at least $50 \%$ of the mass.

\subsection{Warm Clouds, with and without Cool Cores}

Spectra obtained by the Lund team (see Lundstrom et al. 1991) using the ESO 1.4m CAT+CES with reticon detector have outstanding S:N characteristics, with values of up to 1000 , allowing detections of sub-m $\AA$ NaI D lines, ideal for exploring $\mathrm{H}$ column densities below $10^{18} \mathrm{~cm}^{-2}$. A field in Sco-Oph, with several such clouds tagged by radial velocity, is shown in Fig.4. The warm cloud masses range from $1 \mathrm{M}_{\odot}$ to $150 \mathrm{M}_{\odot}$. and only one has a cool core, identified via $\mathrm{KI}$, but this is not a statistically reliable sample. In an overlapping field $10^{\circ}$ north of that in Fig.4 are six cool clouds, masses between $100 \mathrm{M}_{\odot}$ and $600 \mathrm{M}_{\odot}$, two thirds with warm envelopes. This warm cloud identification method, used so far over only a few $\%$ of the sky, has great potential. A well-known diagnostic, the ratio $\mathrm{N}(\mathrm{NaI}) / \mathrm{N}(\mathrm{CaII})$, was first postulated by Routly and Spitzer as showing post-shock gas,given its low value for high LSR velocities in the LISM. We show in Fig.5 this ratio v. N(NaI); all the 


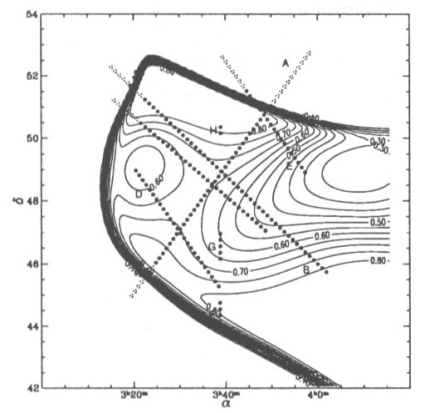

Fig. 2. Isotherm map (units of 50K) of the nearby cool cloud in Perseus, from $21 \mathrm{~cm}$ observations, using neural network methods to compensate a sparse sample (Serra Ricart et. al. 1995). Note the internal temperature minima.

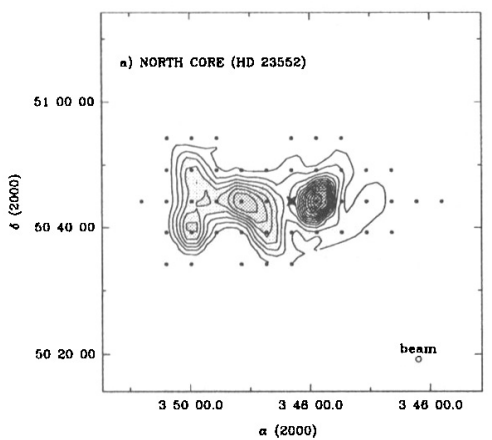

Fig. 3. Integrated CO emissivity over, in units of $0.2 \mathrm{~K} \mathrm{kms}^{-1}$ over a molecular core in the Perseus cloud. The star HD 23552, whose KI LISM absorption gave the original column density and velocity data is shown by a cross.

data is from low velocity clouds, with $\mathrm{V}_{L S R}<30 \mathrm{~km} \mathrm{~s}^{-1}$. Two regimes are seen, and low ratios correspond to low $\mathrm{N}(\mathrm{NaI})$ i.e. to warm, not cool clouds (whose signatures are all in the upper left of the Figure). At low velocity the effect is purely due to temperature difference, i.e. grain evaporation not shocks.

Acknowledgements. This work was carried out with the aid of grant PB94-11-7 of the Spanish DGYCIT. 


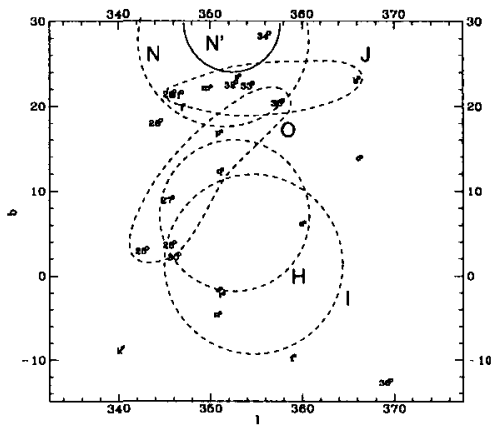

Fig. 4. Warm clouds identified using weak NaI absorption (dashed lines). In the present field only one of these ( $\mathrm{N} \mathrm{N}^{\prime}$ ) has an identified cool core (solid line).

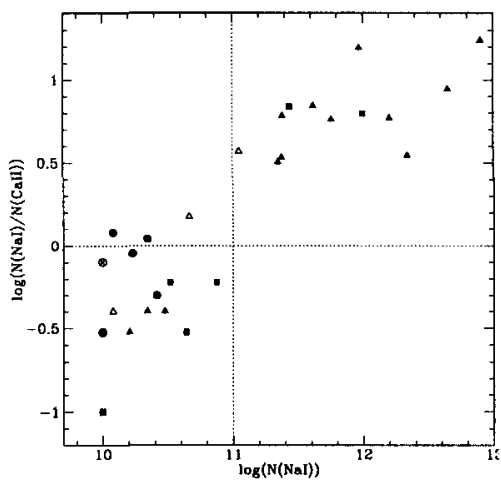

Fig. 5. The $\mathrm{N}(\mathrm{NaI}) / \mathrm{N}(\mathrm{CaII})$ ratio, plotted against $\mathrm{N}(\mathrm{NaI})$, for low velocity clouds in the LISM. Low values for the ratio correspond to low column densities, i.e. to warm ( $\mathrm{T}>5000 \mathrm{~K})$ LISM clouds.

\section{References}

Lundstrom, I., Ardeberg, A., Maurice, E., Lindgren, H. (1991): A\&A 91, 199

Routly, P.M., Spitzer, Jr.L. (1952): ApJ 115, 225

Serra-Ricart, M., Trapero, J., Beckman, J.E., Davies, R.D., Watson, R.A. (1995): AJ 109,312

Trapero, J., Beckman, J.E., Génova, R., McKeith, C.D. (1992): ApJ 394, 552 Trapero, J., Beckman, J.E., Serra-Ricart, M., Davies, R.D., Watson, R.A., García López, R.J. (1995): ApJ 445, 231

Trapero, J. Sempere, M.J., Beckman, J.E., Hobbs, L.M. (1996): ApJ 457, 731 